\title{
Homogeneous vs Heterogeneous Clustered Sensor Networks: A Comparative Study
}

\author{
Vivek Mhatre, Catherine Rosenberg \\ School of Electrical and Computer Eng., Purdue University, West Lafayette, IN 47907-1285 \\ Phone: (765)-494-0034, Fax: (765)-494-0880, Email: \{mhatre, cath\}@ecn.purdue.edu
}

\begin{abstract}
We present a cost based comparative study of homogeneous and heterogeneous clustered sensor networks. We focus on the case where the base station is remotely located and the sensor nodes are not mobile. Since we are concerned with the overall network dimensioning problem, we take into account the manufacturing cost of the hardware as well as the battery energy of the nodes. A homogeneous sensor network consists of identical nodes, while a heterogeneous sensor network consists of two or more types of nodes (organized into hierarchical clusters). We first consider single hop clustered sensor networks (nodes use single hopping to reach the cluster heads). We use LEACH as the representative single hop homogeneous network, and a sensor network with two types of nodes as a representative single hop heterogeneous network. For multi-hop homogeneous networks (nodes use multi-hopping to reach the cluster head), we propose and analyze a multi-hop variant of LEACH that we call M-LEACH. We show that M-LEACH has better energy efficiency than LEACH in many cases. We then compare the cost of multi-hop clustered sensor networks with M-LEACH as the representative homogeneous network, and a sensor network with two types of nodes (that use in-cluster multi-hopping) as the representative heterogeneous network.
\end{abstract}

\section{INTRODUCTION}

Wireless sensor networks have been envisioned to have a wide range of applications in both military as well as civilian domains [1]. Researchers have studied several aspects of wireless sensor networks such as routing, MAC and collaborative data gathering mechanisms ([3], [4], [5], [2], $[8])$. Energy efficiency is a key design objective in most of the research related to wireless sensor networks because the nodes are severely energy constrained, and battery replenishment is often not practical. However besides energy efficiency, hardware complexity is another important aspect that needs to be taken into account when studying the overall network design problem.

We consider clustered sensor networks because clustering allows for scalability of MAC and routing. Cluster heads also serve as fusion points for aggregation of data, so that the amount of data that is actually transmitted to the base station is reduced. Clustered sensor networks can be classified into two broad types; homogeneous and heterogeneous sensor networks.

In homogeneous networks all the sensor nodes are identical in terms of battery energy and hardware complexity. With purely static clustering (cluster heads once elected, serve for the entire lifetime of the network) in a homogeneous network, it is evident that the cluster head nodes will

This work was supported in part by a DARPA grant (contract no. MDA 972-02-1-0032) and an NSF grant (contract no. NSF 0330016). be over-loaded with the long range transmissions to the remote base station, and the extra processing necessary for data aggregation and protocol co-ordination. As a result the cluster head nodes expire before other nodes. However it is desirable to ensure that all the nodes run out of their battery at about the same time, so that very little residual energy is wasted when the system expires. One way to ensure this is to rotate the role of a cluster head randomly and periodically over all the nodes as proposed in LEACH [5]. However the downside of using a homogeneous network and role rotation is that all the nodes should be capable of acting as cluster heads, and therefore should possess the necessary hardware capabilities.

On the other hand, in a heterogeneous sensor network, two or more different types of nodes with different battery energy and functionality are used. The motivation being that the more complex hardware and the extra battery energy can be embedded in few cluster head nodes, thereby reducing the hardware cost of the rest of the network. However fixing the cluster head nodes means that role rotation is no longer possible. When the sensor nodes use single hopping to reach the cluster head, the nodes that are farthest from the cluster heads always spend more energy than the nodes that are closer to the cluster heads. On the other hand when nodes use multi-hopping to reach the cluster head, the nodes that are closest to the cluster head have the highest energy burden due to relaying. Consequently there always exists a non-uniform energy drainage pattern in the network.

Thus there are two desirable characteristics of a sensor network, viz. lower hardware cost, and uniform energy drainage. While heterogeneous networks achieve the former, the homogeneous networks achieve the latter. However both features cannot be incorporated in the same network. The objective of this paper is to compare homogeneous and heterogeneous sensor networks from the point of view of the overall network cost by taking into account the above energy-hardware trade-off.

Clustered sensor networks could also be classified as single hop and multi-hop. A single hop network is one in which sensor nodes use single hopping to reach the cluster head. In a multi-hop network nodes use multi-hopping to reach the cluster head. In both cases, the cluster heads use single hopping to reach the base station, since we assume a remote base station. We first compare the costs of homogeneity versus heterogeneity in a single hop network, and then in a multi-hop network. For a multi-hop network, we 
propose and analyze a data gathering scheme that we call M-LEACH or Multi-hop LEACH (since LEACH is single hop).

The outline of the paper is as follows. In section II, we briefly discuss a representative single hop homogeneous and a representative single hop heterogeneous network. Section III contains analytical and numerical results about the cost based comparison between these two networks. In section IV, we propose and study an extension of LEACH that we call M-LEACH. Comparison between multi-hop homogeneous and heterogeneous networks is studied in section $\mathrm{V}$. Finally we conclude in section VI.

\section{Single Hop Networks}

In a single hop network, the sensor nodes communicate directly with the cluster head using a single hop transmission. The nodes are assumed to have power control features so as to adjust their transmit power.

\section{A. Single Hop Homogeneous Network: LEACH}

Following are some of the salient features of a single hop homogeneous sensor network.

- Since all the nodes are identical, the main design objective is to guarantee a certain network lifetime (in terms of number of data gathering cycles), and at the same time ensure that all the nodes expire at about the same time so that there is very little residual energy left behind when the network expires. Hence LEACH uses random and periodic rotation of the cluster heads for load balancing. Role rotation also ensures that a node which is located near the periphery of a cluster is nearer to the cluster head at some other time.

- Since each node has to be capable of acting as a cluster head, it is necessary for each node to have the hardware capable of performing long range transmissions to the remote base station, complex data computations (if required), and co-ordination of MAC and routing within a cluster.

- Since all the nodes are capable of acting as a cluster head, the failure of a few nodes does not seriously affect the working of the scheme. Thus the system is robust to node failures.

\section{B. Single Hop Heterogeneous Networks: Two Types of Nodes}

Heterogeneous sensor networks use two or more types of nodes with different functionalities. For example, the authors in $[6]$ propose using two types of nodes; type 0 nodes which act as pure sensor nodes, and type 1 nodes which act as the cluster head nodes. Some of the salient features of such networks are:

- Since the cluster head nodes are predetermined, and the sensor nodes use single hop communication to reach the cluster head nodes, the sensor nodes near the periphery of the cluster have the highest energy expenditure among all the sensor nodes. It is this worst case energy expenditure that has to taken into account in battery energy dimensioning. Thus there is a waste of energy due to the residual battery energy of the sensor nodes that are near the cluster heads.

- Since only the cluster head nodes bear the responsibility of transmitting to the distant base station, the rest of the nodes can be designed with simple hardware that enables short range communication. Thus the hardware complexity is limited to only a few nodes.

- A cluster head node serves as the fusion point, as well as the command center of its cluster. As a result when a cluster head node fails, all the sensor nodes in that cluster have to be re-assigned to other neighboring clusters. In the extreme case, it is possible that all the cluster head nodes might fail, thereby bringing down the entire network. Thus the system is less robust to node failure as compared to a homogeneous sensor network.

\section{Cost-Based Comparison of Single Hop Homogeneous and Heterogeneous Networks}

Consider a sensor network in which $n_{0}$ sensor nodes are deployed over a circular region of radius $A$. The number of sensor nodes $n_{0}$ is determined by the application and sensing coverage requirements. We assume that $n_{0}$ is fixed and is given to us. A remote base station is located at distance $d$ from the center of the region. The nodes are organized in clusters based on their proximity from the cluster head nodes. We assume a discrete data gathering model in which during each data gathering cycle, every node sends a packet to the cluster head. We also assume the aggregation model in [5] in which the cluster head node collects all the packets from the nodes in its cluster, and aggregates them into a single packet that is sent to the base station. The energy spent on transmitting a packet over distance $x$ within a cluster is $l_{1}+\mu_{1} x^{k_{1}}$, while the energy required to transmit a packet over distance $d$ from a cluster head to the base station is $l_{2}+\mu_{2} d^{k_{2}}$. The amount of energy spent in the transmitter electronics circuitry during the packet transmission is modeled by $l_{i}$, while $\mu_{i} x^{k}$ is the energy spent in the RF power amplifier to counter the propagation loss. In general we would expect $k_{2}>k_{1}$ since the in-cluster communication is likely to be line-of sight, while the communication between the cluster heads and the remote base station is likely to be multi-path [9]. Let the amount of energy spent on aggregating $n$ packets at the cluster head node be $n E_{f}$.

\section{A. Summary of Results on LEACH}

In the experimental settings of LEACH, the authors take $k_{1}=2$ and $k_{2}=4$. Let $m_{1}$ be the optimum number of cluster head nodes for these settings (as given by Eq. (19) in $[5])^{1}$.

$$
m_{1}=\frac{\sqrt{n_{0}}}{\sqrt{2}} \sqrt{\frac{\mu_{1}}{\mu_{2}}} \frac{A}{d^{2}}
$$

Let $E$ be the desired battery energy of each of the $n_{0}$ nodes required for sustaining $T$ data gathering cycles. We have

\footnotetext{
${ }^{1}$ Note that the above expression does not have a $\sqrt{\pi}$ term in the denominator as compared to the original expression in [5], because that expression was derived for a square region, while we consider a circular region.
} 
assumed a model of the sensor network where data is gathered in discrete cycles, and our objective is to guarantee $T$ such data gathering cycles. Eq. (18) in [5] is the expression for the overall energy spent in the network by all the nodes during each data gathering cycle. Since role rotation ensures that the overall energy expenditure of the network is shared equally by all the nodes, the energy expenditure of each node during each cycle can be obtained by dividing the above expression by $n_{0}$. Furthermore, if it is desired that the system last for at least $T$ data gathering cycles, then we should further multiply the above expression by $T$. Thus we obtain:

$$
E=T\left(2 l_{1}+E_{f}+\frac{m_{1} \mu_{2} d^{4}}{n_{0}}+\frac{\mu_{1} A^{2}}{2 m_{1}}\right)
$$

Note that in order to ensure effective role rotation, $T$ should be high enough (at least comparable to $n_{0}$ ) so that each node is elected as a cluster head at least once. In fact the way the authors of LEACH implement the scheme is by breaking up $T$ cycles into several rounds, each consisting of several cycles. In each round new cluster head nodes are elected.

\section{B. Summary of Results on a Single Hop Heterogeneous Network}

The authors in [6] propose using $n_{1}$ cluster head nodes which have a higher battery energy, and a more complex hardware than the sensor nodes. This is in addition to the $n_{0}$ sensor nodes. The authors model the cost of a node as the sum of its hardware cost, and its battery cost. Hence the cost of a sensor node is $\alpha_{0}+\beta E_{0}$ where $\alpha_{0}$ takes into account the hardware cost of the node, $E_{0}$ is the battery energy of the node, and $\beta$ is the proportionality constant for the battery cost. Similarly the cost of a cluster head node is $\alpha_{1}+\beta E_{1}$. The optimum number of cluster heads, $n_{1}$, is given by Eq. (19) in [6] with $k=2$,

$$
n_{1}=\sqrt{\frac{n_{0} \beta T \mu_{1} A^{2}}{\alpha_{1}+\beta T\left(l_{2}+\mu_{2} d^{4}\right)}}
$$

Similarly, the required battery energies of the two types of nodes $\left(E_{1}, E_{0}\right)$ are given by Eq. (15) and Eq. (16) in [6]:

$$
\begin{aligned}
& E_{1}=T\left(\frac{n_{0}}{n_{1}}\left(l_{1}+E_{f}\right)+\left(l_{2}+\mu_{2} d^{4}\right)\right) \\
& E_{0}=T\left(l_{1}+\frac{\mu_{1} A^{2}}{n_{1}}\right)
\end{aligned}
$$

\section{Cost Analysis}

Let $f_{1}\left(\alpha_{0}, \alpha_{1}, \beta\right)$ denote the overall network cost when LEACH is used, i.e., the cost of the homogeneous network, and let $f_{2}\left(\alpha_{0}, \alpha_{1}, \beta\right)$ denote the network cost when two types of nodes are used, i.e., the cost of the heterogeneous network. Since each node in LEACH has hardware capable of acting as a cluster head, the hardware cost of each node in LEACH is $\alpha_{1}$. Hence we obtain:

$$
\begin{aligned}
& f_{1}\left(\alpha_{0}, \alpha_{1}, \beta\right)=n_{0}\left(\alpha_{1}+\beta E\right) \\
& f_{2}\left(\alpha_{0}, \alpha_{1}, \beta\right)=n_{0}\left(\alpha_{0}+\beta E_{0}\right)+n_{1}\left(\alpha_{1}+\beta E_{1}\right)
\end{aligned}
$$

\begin{tabular}{|l|l|l|}
\hline & Scenario I & Scenario II \\
\hline No. of sensor nodes, $n_{0}$ & 100 & $10^{5}$ \\
\hline Radius of the region, $A$ & $56 \mathrm{~m}$ & $1000 \mathrm{~m}$ \\
\hline $\begin{array}{l}\text { Distance from } \\
\text { the base station, } d\end{array}$ & $125 \mathrm{~m}$ & $2000 \mathrm{~m}$ \\
\hline Length of each packet & 525 bytes & 525 bytes \\
\hline$l_{1}=l_{2}$ & $0.21 \mathrm{~mJ}$ & $0.21 \mathrm{~mJ}$ \\
\hline$\mu_{1}$ & $42 \mathrm{~nJ} / \mathrm{m}^{2}$ & $5.46 \mathrm{pJ} / \mathrm{m}^{4}$ \\
\hline$\mu_{2}$ & $5.46 \mathrm{pJ} / \mathrm{m}^{4}$ & $5.46 \mathrm{pJ} / \mathrm{m}^{4}$ \\
\hline$k_{1}$ & 2 & 4 \\
\hline$k_{2}$ & 4 & 4 \\
\hline$E_{f}$ (per packet) & $0.021 \mathrm{~mJ}$ & $0.021 \mathrm{~mJ}$ \\
\hline
\end{tabular}

TABLE I

SYSTEM PARAMETERS

Let $f\left(\alpha_{0}, \alpha_{1}, \beta\right)$ be the difference between the above two costs. Substituting for $E, E_{0}$ and $E_{1}$ from (2), (5) and (4), and after some simplifications, we obtain,

$$
\begin{aligned}
f\left(\alpha_{0}, \alpha_{1}, \beta\right)= & f_{1}\left(\alpha_{0}, \alpha_{1}, \beta\right)-f_{2}\left(\alpha_{0}, \alpha_{1}, \beta\right) \\
= & n_{0}\left(\alpha_{1}-\alpha_{0}\right)-n_{1} \alpha_{1}+\beta T\left(m_{1}-n_{1}\right) \mu_{2} d^{4}+ \\
& \beta T\left(n_{0} \mu_{1} A^{2}\left(\frac{1}{2 m_{1}}-\frac{1}{n_{1}}\right)-n_{1} l_{2}\right)
\end{aligned}
$$

Note that in the above expression for $f(),. n_{1}$ is a function of $\alpha_{1} / \beta$ (see (3)). Thus we obtain an expression for the cost difference between a homogeneous and a heterogeneous network as a function of the manufacturing cost parameters $\alpha_{i}$ and $\beta$.

\section{Case Studies}

Table I summarizes the settings that we consider for the case studies. The transceiver parameters are similar to those of the state-of-the art transceivers that are currently available (see [5]). Also note that in our analysis $l_{1}, l_{2}$, $\mu_{1}$ and $\mu_{2}$ are per packet quantities that are obtained by multiplying the corresponding per bit quantities in [5] by the packet size. In this section we use scenario I for cost based comparison of single hop networks, while scenario II is used for cost based comparison of multi-hop networks in section $\mathrm{V}$. The reason being that multi-hop networks are more cost effective than single hop networks when the size of the region is large, and the propagation fall-off exponent for in-cluster communication is high $\left(k_{2}=4\right)$. This argument will be validated through numerical results in section IV.

For scenario I in Table I the dimensions of the region $(A$, d) are chosen to closely resemble the LEACH settings in [5]. We consider a circular region of radius $56 \mathrm{~m}$ so as to have the same area as in LEACH where the authors consider a $100 \mathrm{~m} \times 100 \mathrm{~m}$ square region. The number of nodes is the same, i.e., 100 as in the simulation settings of LEACH.

We plot the difference in the costs of LEACH and the heterogeneous single hop network as a function of $\alpha_{1} / \beta E$ for various values of $\alpha_{0}$, where $E$ is given by (2). For example $\alpha_{0}=0.1 \alpha_{1}$ implies that the hardware cost of a cluster 


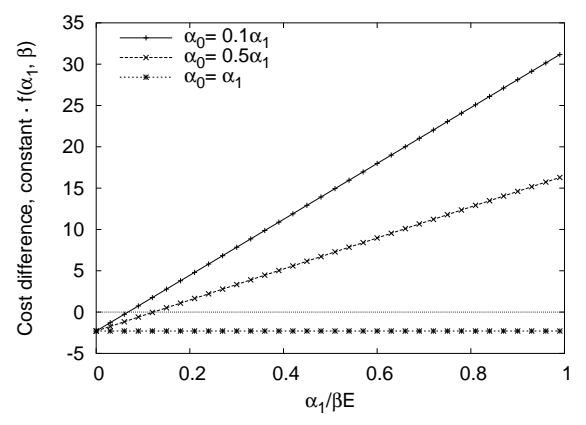

Fig. 1. Scenario I: Difference in the cost of the homogeneous $(\mathrm{LEACH})$ and the heterogeneous network (two types of nodes), $f($. as a function of the ratio of the hardware cost $\left(\alpha_{1}\right)$ to the battery $\operatorname{cost}(\beta E)$. Nodes use single hopping within the cluster.

head node is much more than that of a sensor node. On the other hand, $\alpha_{0}=\alpha_{1}$ implies that there is no additional hardware cost of adding cluster head functionality to a sensor node. Since $E$ (Eq. (2)) is the battery energy required for each node in LEACH, it does not depend on $\alpha_{i}$ or $\beta$. We use $\alpha_{1} / \beta E$ as the $\mathrm{x}$-axis for the plots of the cost difference because it is an indicator of the relative cost of the hardware and the battery energy.

In Figure 1 when the curve is above the zero line, the homogeneous network (LEACH) costs more than the heterogeneous network and vice versa. First we note that when $\alpha_{1}=\alpha_{0}$, the homogeneous network is more cost effective than the heterogeneous network, since we do not gain anything by embedding all the functionality of a cluster head in a few nodes. We also observe that the cost difference between the two networks increases as the relative cost of the hardware as compared to the battery cost increases. This is something we would expect, since $\mathrm{LEACH}$ requires complex hardware in all the nodes, and as the cost of the hardware increases, the overall network cost of LEACH increases. This increase is linear in $\alpha_{1} / \beta$ because the expression for $f($.$) in (8) is linear in \alpha_{1}$, and even though $n_{1}$ is a function of $\alpha_{1} / \beta$, for the range of values of $\alpha_{1} / \beta E$ that we consider in Figure 1, $n_{1}$ is almost a constant. We also note that when $\alpha_{1}=\alpha_{0}=0$ (points on y-axis), LEACH outperforms the heterogeneous network. This is because the hardware cost of the nodes simply does not figure in the overall network cost. As a result, the homogeneous network costs less in terms of energy due to its uniform energy drainage pattern.

We also observe that for the above range of values of $\alpha_{1} / \beta E$ the required number of cluster heads for the homogeneous network is 2 , while for the heterogeneous network is 3 (given by (1) and (3)). The reason for such low number of cluster heads is that for the settings that we consider, the propagation constant for communication between the cluster heads and the base station is very high $\left(k_{2}=4\right)$, and the distance of the remote base station from the region of interest is large. As a result, direct transmissions to the base station are energy intensive, and hence the optimum solution favors fewer cluster heads.

Since the number of cluster heads is small, most of the system complexity of a heterogeneous network is embedded in a small number of cluster head nodes. Consequently when these nodes fail, the system stops functioning. On the other hand a system based on LEACH is more resilient to node failures, since every node is capable of acting as a cluster head.

\section{Multi-Hop LEACH (M-LEACH)}

In this section we propose an extension to LEACH. The system parameters that the authors consider in [5] such as the size of the region, the distance of the base station, the radio characteristics of the transceiver etc. are suited for the data gathering scheme that they propose. However, we note that in general using single hop communication within a cluster for communication between the sensor nodes and the cluster heads may not be the optimum choice. This is particularly the case when the propagation loss index for in-cluster communication is large $\left(k_{1}>2\right)$. This is likely to be the case when the sensor nodes are deployed in regions of dense vegetation or uneven terrain. In such cases, it may be beneficial to use multi-hop communication among the nodes in the cluster to reach the cluster head. This is the motivation behind M-LEACH.

We assume that all the nodes use a communication radius of $R$ for in-cluster communication. We minimize the overall network energy expenditure with respect to two variables, viz. $R$ and the size (i.e. the radius) of each cluster $a$ (which depends on the number of cluster heads). As in LEACH, the cluster head nodes are rotated periodically and randomly. This ensures that the following two sets of nodes are relieved of their energy burdens:

1. The cluster head nodes which have to perform long range transmissions to the distant base station.

2. The nodes which are within one hop of the cluster head nodes (henceforth referred to as critical relay nodes) which have the highest burden of relaying of packets within a cluster.

Thus a randomized periodic role rotation ensures that all the nodes are equally likely to be cluster head nodes, as well as critical relay nodes. If in the optimum solution, we obtain that $R=a$, then this implies that single hopping for in-cluster communication is more energy efficient than multi-hopping. In this single hopping scheme the nodes use the same power level, since they have the same communication radius $R$. However the overall energy expenditure can be further reduced if nodes use power control, and this scheme is nothing but LEACH. On the other hand if we obtain that $a>R$, then the nodes must use multi-hop communication, and the quantity $a / R$ is a good indicator of the hop count. Note that studying a multi-hopping system with power control (instead of assuming a fixed communication radius $R$ ) is much more difficult to analyze both in terms of energy expenditure and connectivity.

\section{A. Problem Formulation}

Consider the same system scenario as discussed in Section III. Nodes are organized as clusters, and they use multi-hop communication within the cluster to reach the 
cluster head node. The cluster head node aggregates the received data, and transmits it to the remote base station. As in LEACH, the cluster head nodes are rotated randomly and periodically for load balancing. Let there be $m$ clusters. Since the cluster heads are chosen randomly, we assume that the clusters are uniformly distributed over the entire region, and each cluster on an average has a radius of $A / \sqrt{m}$.

All the nodes use a common communication radius of $R$. Without loss of generality we assume that $R \leq A / \sqrt{m}$ where the equality corresponds to single hop communication within the cluster, while the inequality corresponds to multi-hop communication. In order that multi-hop communication be possible, it is necessary that $R$ be large enough so that connectivity of nodes is guaranteed with a high probability. As in [6], [7], we require that $R>r$ where a communication radius of $r$ ensures node connectivity with a probability of at least $1-\epsilon$ for a given $\epsilon$.

$$
r=A \sqrt{\frac{1}{n_{0}} \log \left(\frac{n_{0}}{\epsilon}\right)}
$$

We also assume perfect synchronization of MAC so that there is no energy wasted on IDLE listening. This assumption is valid for data gathering sensor networks where data gathering phases are discrete, and the cluster head can be assumed to be in charge of MAC so that IDLE listening is minimized. The assumption of ideal MAC can be relaxed later to take into account a particular MAC scheme, however the overall approach to solving the problem does not change. Besides, the above approach provides benchmark results with an ideal MAC in mind. From now on, we assume this idealized MAC scheme.

Consider a typical cluster. As in [6], we can divide the circular cluster into concentric rings of thickness $R$. Let $a=A / \sqrt{m}$ be the radius of the cluster. Since aggregation is performed only at the cluster heads, we can determine the total energy spent during each data gathering cycle. Each node has to relay a certain number of packets based on where it is located, and also has to transmit its own packet. The number of packets that enter the $n$th ring from outside the ring is simply $N_{n}=n_{0}\left(a^{2}-n^{2} R^{2}\right) / A^{2}$. Hence the nodes in the $n$th ring spend $N_{n}\left(2 l_{1}+\mu_{1} R^{k_{1}}\right)$ amount of energy on relaying of packets. In addition to this, each node has to transmit its own packet, and the cluster head has to perform a single long range transmission to the base station. Hence if we denote $P(R, m)$ to be the total amount of energy spent in the network during a data gathering cycle, then we obtain:

$$
\begin{aligned}
& P(m, R)=m\left(l_{2}+\mu_{2} d^{k_{2}}\right)+n_{0}\left(l_{1}+\mu_{1} R^{k_{1}}\right)+ \\
& m\left(\frac{n_{0}}{m}\right)\left(l_{1}+E_{f}\right)+ \\
& m\left\{\sum_{n=1}^{a / R}\left(2 l_{1}+\mu_{1} R^{k_{1}}\right) n_{0} \frac{\left(a^{2}-n^{2} R^{2}\right)}{A^{2}}\right\}
\end{aligned}
$$

In the above expression, the first term corresponds to the energy spent on transmission from the cluster heads to the

\begin{tabular}{|l|l|l|l|l|}
\hline Scenario I & $\mathrm{m}$ & $\mathrm{P}$ & $\mathrm{a}$ & $\mathrm{R}$ \\
\hline LEACH & 2 & $5 \times 10^{-4} \mathrm{~J}$ & - & - \\
\hline M-LEACH & 3 & $6.78 \times 10^{-4} \mathrm{~J}$ & 32.8 & 32.8 \\
\hline \multicolumn{5}{|c}{ TABLE II } \\
NUMERICAL RESULTS FOR SCENARIO I
\end{tabular}

base station, the second term corresponds to the energy spent by all the nodes on transmitting their own packet, the third term represents the energy spent by the cluster head nodes on receiving and aggregating packets, and the last term is the summation of the energy spent on relaying packets over all the rings of the cluster. We would like to determine the optimum values of $m$ and $R$ which minimize $P(m, R)$ subject to the connectivity constraint $R \geq r$.

\section{B. Problem Solution}

Let $\theta=\left(l_{2}+\mu_{2} d^{k_{2}}\right)$. After some simplification of (10) we obtain the following expression for $P(a, R)$, where we use $m=A^{2} / a^{2}$ to eliminate $m$.

$$
\begin{aligned}
P(a, R)= & \frac{\theta A^{2}}{a^{2}}+n_{0}\left(2 l_{1}+E_{f}+\mu_{1} R^{k_{1}}\right) \\
& +\frac{n_{0}\left(2 l_{1}+\mu_{1} R^{k_{1}}\right)(4 a+R)(a-R)}{6 a R}
\end{aligned}
$$

Our objective is to minimize the above expression as a function of $a$ and $R$ with (9) as a constraint. It is not possible to find a closed form expression for $R$ and $a$ using this approach. Hence we require numerical methods to solve the minimization problem for a given set of network parameters. We compare the obtained results with LEACH. Our criterion for comparison between M-LEACH and LEACH is the average energy per node per cycle, i.e., $P=E / T$. This is because in terms of hardware complexity, both are similar, since they require each node to act as a cluster head. We provide comparative results between LEACH and M-LEACH to support our argument that when the propagation constant is large, and the size of the region is large, instead of using the LEACH approach of single hopping, using multi-hopping within a cluster could prove more energy efficient. In Tables II and III, $m$ denotes the optimum number of cluster heads, while $P$ denotes the average energy expenditure of a node during each data gathering cycle and equals $E / T$.

For the system parameters as given in Table I scenario I, we obtain that for M-LEACH $a=R$ (see Table II). This indicates that although we start with a multi-hop network, the optimum solution consists of a single hop network. Since M-LEACH does not use power control for in-cluster communication, it performs worse in terms of energy than LEACH when it comes to pure single hop network. This is reflected in the energy term in Table II. However when the optimum solution of M-LEACH is more in favor of multihop, i.e., $a>R$, then M-LEACH out-performs LEACH. This is especially true when the propagation loss exponent is large, or the size of the region is large. This is precisely the case for scenario II. 


\begin{tabular}{|l|l|l|l|l|}
\hline Scenario II & $\mathrm{m}$ & $\mathrm{P}$ & $\mathrm{a}$ & $\mathrm{R}$ \\
\hline LEACH & 16 & $21.53 \times 10^{-3} \mathrm{~J}$ & - & - \\
\hline M-LEACH & 2 & $5.73 \times 10^{-3} \mathrm{~J}$ & $707 \mathrm{~m}$ & $70 \mathrm{~m}$ \\
\hline
\end{tabular}

TABLE III

NumericAl RESUlts FOR SCENARIO II

The solution to (11) for scenario II from Table I is given in Table III. Since for scenario II, $k_{1}=k_{2}=4$, we cannot directly use the results from (1) and (2) for LEACH, since those were derived by assuming $k_{1}=2$ and $k_{2}=4$. However it is easy to generalize those results for any $k_{1}$ and $k_{2}$. The values of $m$ and $E$ for LEACH in scenario II were obtained by appropriately re-deriving the expressions for $m$ and $E$ for $k_{1}=4$ and $k_{2}=4$. We note in Table III that since for M-LEACH $R$ is smaller than $a$, i.e., the communication radius is smaller than the cluster radius, multi-hop mode is the optimum mode of communication. We also note from Table II that the optimum communication radius $R$ is greater than the minimum communication radius $r$ required for connectivity, since to ensure connectivity with a probability of at least 0.99 , we obtain $r=13 \mathrm{~m}$. Thus for scenario II M-LEACH clearly out-performs LEACH.

\section{Multi-Hop Networks}

Having obtained results for M-LEACH, we use the results for the cost of a multi-hop heterogeneous network that have already been obtained in [6], and then we can determine the corresponding cost-difference function $f($.$) as in$ (8). We consider the settings of scenario II for this comparison, because the high propagation loss for in-cluster communication, and large size of the region favor multihopping. The approach for cost-based comparison is exactly similar to the single hop case. In this case we consider M-LEACH as the representative multi-hop homogeneous network as we have already seen in the previous section that for scenario II, M-LEACH is more energy efficient than LEACH. We consider a network with two types of nodes as the representative heterogeneous network. For this network and for the settings of scenario II, we obtain that the optimum radius of communication is $\hat{R}=93 \mathrm{~m}$, while for M-LEACH the optimum radius of communication is $70 m$ (see Table III). We plot the cost difference between the two multi-hop networks in Figure 2. In this case, $E$ in $\alpha_{1} / \beta E$ along the $\mathrm{x}$-axis is the battery energy of M-LEACH. Again the nature of the plot is similar to that of Figure 1. As the relative cost of the hardware increases, the heterogeneous network is more cost effective than the homogeneous network. We also observe that for the range of $\alpha_{1} / \beta E$ that we consider, M-LEACH performs much better than its heterogeneous counterpart (Figure 2) as compared to the performance of LEACH versus its heterogeneous counterpart (Figure 1). This is especially the case when $\alpha_{0}=0.5 \alpha_{1}$, where M-LEACH out-performs the heterogeneous multi-hop network. Thus in general to determine the most cost effective solution (homogeneous or heterogeneous, single hop or multi-hop), we must compare

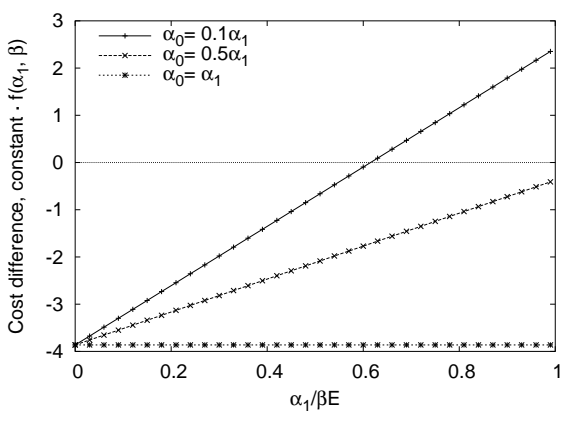

Fig. 2. Scenario II: Difference in the cost of the homogeneous (MLEACH) and the heterogeneous network (two types of nodes), $f($. as a function of the ratio of the hardware cost $\left(\alpha_{1}\right)$ to the battery $\operatorname{cost}(\beta E)$. Nodes use multi-hopping within the cluster.

the overall network cost.

\section{Conclusion}

We first presented a cost based comparative analysis of single hop homogeneous and single hop heterogeneous networks. We took into account the hardware as well as the battery cost of the nodes in our analysis. We also proposed and analyzed a generalization of LEACH called M-LEACH (Multi-hop LEACH) which uses multi-hop communication within the cluster, and uses random and periodic cluster head rotation for load balancing. We showed that in many cases M-LEACH is more energy efficient than LEACH. Using M-LEACH as the representative multi-hop homogeneous network, we presented a cost based comparison of multi-hop homogeneous and multi-hop heterogeneous networks.

\section{REFERENCES}

[1] I.F. Akyildiz, W. Su, Y. Sankarsubramaniam and E. Cayirci, "Wireless Sensor Networks: a survey", Computer Networks, Vol. 38, pp. 393-422, March 2002.

[2] S. Bandyopadhyay and E. Coyle, An Energy Efficient Hierarchical Clustering Algorithm for Wireless Sensor Networks, IEEE Infocom, San Francisco, CA, April 2003.

[3] C. Intanagonwiwat, R. Govindan, D. Estrin, J. Heidemann, and F. Silva. "Directed Diffusion for Wireless Sensor Networking", ACM/IEEE Transactions on Networking, 11 (1 ), pp. 2-16, February, 2002.

[4] S. Lindsey, C. Raghavendra, "PEGASIS: Power Efficient GAthering in Sensor Information Systems", 2002 IEEE Aerospace Conference, March 2002.

[5] W. Heinzelman, A. Chandrakasan and H. Balakrishnan. "An Application-Specific Protocol Architecture for Wireless Microsensor Networks," IEEE Transactions on Wireless Communications, Vol. 1, No. 4, October 2002.

[6] V. Mhatre and C. Rosenberg, "Design Guidelines for Wireless Sensor Networks: Communication, Clustering and Aggregation", to appear in Adhoc Networks Journal, Elsevier Science. Available from http://web.ics.purdue.edu/ mhatre/adhoc.pdf.

[7] P. Gupta and P. R. Kumar, "Critical power for asymptotic connectivity in wireless networks," Stochastic Analysis, Control, Optimization and Applications: A Volume in Honor of W. H. Fleming (W M. McEneany, G. Yin and Q. Zhang, eds.), Boston, MA; Birkhauser, 1998. ISBN 0-8176-4078-9.

[8] V. Mhatre, C. Rosenberg, D. Kofman, R. Mazumdar and N. Shroff, "A Minimum Cost Surveillance Sensor Network with a Lifetime Constraint," submitted March 2003. Available from http://web.ics.purdue.edu/ ${ }^{\text {mhatre/lifetime.pdf }}$

[9] T. S. Rappaport, Wireless Communication, Prentice-Hall, 1996. 